\title{
Orientaciones para la intervención logopédica con niños bilingües
}

\author{
Silvia Nieva \\ Universidad Complutense de Madrid y Universidad de Castilla-La Mancha, España
}

\begin{abstract}
Resumen
Los crecientes movimientos migratorios y las demandas sociales de manejo de lenguas para el desarrollo personal y profesional han aumentado el interés por la educación bilingüe. En el Estado Español encontramos un apoyo institucional emergente para la implantación de programas bilingües en lenguas extranjeras así como en lenguas cooficiales y dialectos regionales. Las investigaciones recientes destacan el bilingüismo como una opción que beneficia el desarrollo del lenguaje y de la cognición. Sin embargo, en población que presenta alteraciones del lenguaje aún quedan muchas cuestiones por resolver. Los profesionales se enfrentan a una gran variedad de contextos y situaciones bilingües y la información sobre desarrollo típico, atípico y sobre la efectividad de diferentes modelos de tratamiento es escasa. La presente revisión trata de organizar la información disponible sobre desarrollo y sobre evaluación e intervención basada en la evidencia para orientar a los profesionales en la toma de decisiones y en el asesoramiento a familias de niños con dificultades del habla y el lenguaje en entornos bilingües.
\end{abstract}

Palabras clave: Alteraciones del lenguaje; Bilingüismo; Logopedia; Intervención logopédica; Multilingüismo; Práctica Basada en la Evidencia.

\section{Speech-therapy intervention guidelines for bilingual children}

\begin{abstract}
Growing migration patterns, along with social demands for the use of languages in order to develop both personally and professionally have led to an increasing interest in bilingual education. The Spanish State is one source of emerging institutional support for the implementation of bilingual programs in foreign languages and support for the teaching of co-official languages and regional dialects. Recent investigations claim that bilingualism benefits language development and cognition. However, many questions are still to be resolved in populations that present language disorders. Professionals are faced with a great variety of contexts and bilingual situations, while information on typical and atypical development, and the efficacy of different treatment models is scarce. This review aims to organise the available development-based and evidence-based information so as to provide guidelines to professionals when it comes to taking decisions and counselling families with children who have speaking and language difficulties in bilingual settings.
\end{abstract}

Key words: Speech Therapy; Bilingualism; Multilingualism; Evidence-based Practice; Speech-sound disorders; Speech-therapy intervention.

Correspondencia con los autores: silnieva@ucm.es

Recibido 6 de Abril 2015. Revisado 26 Mayo 2015. Aceptado 9 de Septiembre de 2015 


\section{Introducción}

En los últimos años, el bilingüismo ha despertado un interés creciente entre los investigadores. A pesar de que el 50\% de los niños del mundo crecen expuestos a dos o más lenguas, la mayor parte de la investigación trata sobre el desarrollo en niños monolingües (Grosjean, 2010). Esta tendencia parece estar revirtiéndose en paralelo al desarrollo de diferentes métodos de investigación (Hoff y Rumiche, 2011). Históricamente, la influencia del bilingüismo en el aprendizaje y en el desarrollo lingüístico ha sido considerada negativa. A partir de los años 60 empiezan a obtenerse resultados valorados como positivos (Skuntnab-Kangas, 1981). Para comprender este fenómeno es preciso conocer su realidad más allá de nuestras fronteras.

La diversidad lingüística mundial es un hecho. La decimoctava edición del Ethnologue informa de la existencia en la actualidad de 7.102 lenguas vivas en el mundo, de las cuales 578 tienen reconocimiento institucional (Paul, Simons y Fenning, 2015). La mayor parte se consideran "en desarrollo". Esto no impide que en muchas zonas del mundo las personas hablen con frecuencia más de una lengua en su comunidad, independientemente del nivel de alfabetización. Según esta fuente, Europa es el continente con menor diversidad lingüística, donde existen únicamente 286 lenguas vivas, 73 de ellas institucionales.

En el Ethnologue se distingue entre el número de hablantes de una lengua como segunda lengua dentro de un país y el número total de hablantes de esa misma lengua (p.ej. los hablantes de lengua inglesa en España y el total de hablantes de lengua inglesa en todo el mundo). Esta diferencia se observa claramente en zonas como Europa, donde la situación de bilingüismo es promovida frecuentemente por las instituciones y requiere del aprendizaje de lenguas no utilizadas en el territorio en el que se estudian, como es el caso de la enseñanza del inglés en las escuelas, cuyo alto valor político o estratégico aumenta el número de hablantes fuera de los territorios de uso natural.

No todas las lenguas habladas en un país están reconocidas por su gobierno. Esta situación puede relacionarse con diferentes actitudes hacia las lenguas, así como con su estatus socioeconómico y las implicaciones de ser hablantes plurilingües (competentes en más de dos lenguas). Mientras que en países como Papúa Nueva Guinea se hablan 839 lenguas, 43 de ellas institucionales, en otros países como Australia se encuentran 212 lenguas vivas, 144 en proceso de extinción y únicamente 6 institucionales. Esta situación ejemplifica cómo las políticas lingüísticas pueden influir en la supervivencia de las distintas lenguas del planeta. 
Si nos centramos en el Estado Español, encontramos 15 lenguas y dialectos listados. Entre estas lenguas, las institucionalizadas como oficiales son cinco: castellano, catalán, euskera, gallego y valenciano. Las lenguas de signos reconocidas son la lengua de signos española, la lengua de signos catalana y la lengua de signos valenciana.

\section{Bilingüismo y situaciones bilingües}

Los investigadores que se centran en el estudio del bilingüismo tienen como primera dificultad la definición de su objeto de estudio. Actualmente no existe un acuerdo en la comunidad investigadora sobre qué es el bilingüismo o qué es un entorno bilingüe.

A grandes rasgos, el bilingüismo está relacionado con la competencia en dos lenguas, ocasionalmente en más de dos (Conboy y Khul, 2011). Para comprender este concepto es esencial distinguir entre la lengua que se utiliza en el entorno inmediato (primera lengua o L1 y segunda lengua o L2) y la lenguas extranjeras (LE), que son lenguas de comunicación habitual fuera del país. El contraste entre estos dos tipos de lenguas tiene como base su modo de aprendizaje. La L2 puede ser una de las lenguas familiares del bilingüe y/o la lengua de escolarización, o una lengua aprendida después de la infancia. Su aprendizaje es predominantemente no formal e implícito. Por el contrario, la LE es una lengua no utilizada en el ambiente inmediato y cuyo aprendizaje suele ser explícito e institucionalizado.

Por otra parte, la bibliografía muestra gran variedad terminológica, como puede verse en las distintas denominaciones en inglés para los niños que crecen en entornos bilingües: dual language learners (Bialystok, 2011), bilingual first learners (Hammer et al., 2014) o bilingual developing children (Williams y McLeod, 2012), entre otras.

En relación con el término de bilingüismo, es posible encontrar definiciones más o menos precisas o abiertas en las que se consideran diferentes variables, y que pueden incluir otros canales de comunicación distintos del oral o toleran varios niveles de competencia. Por ejemplo, la definición propuesta por Kohnert (2010, p. 457) pone el foco en variables temporales y contextuales: "los niños bilingües se definen de forma operativa como aquellos individuos que reciben un input regular en dos o más lenguas durante el periodo más dinámico del desarrollo comunicativo - en algún momento entre el nacimiento y la adolescencia (...) esta definición incluye a niños que aprenden dos lenguas desde el nacimiento, así como aquellos que aprenden una primera lengua al nacer y empiezan con una segunda lengua en la escuela", mientras que la propuesta de 
Grech y McLeod (2012, p.121) para el término multilingüismo, que incluye la definición de bilingüismo, está centrada en variables de procesamiento de la información: "una persona multilingüe es capaz de comprender o producir dos o más lenguas en forma oral, manual o escrita, independientemente del nivel de competencia o uso y de la edad en que las lenguas fueron aprendidas".

Como puede observarse, existe una tendencia en estos casos a asimilar el término bilingüismo al de plurilingüismo o multilingüismo. No obstante, estos dos términos no están exentos de ambigüedad. En el Marco Común Europeo de Referencia para las Lenguas se consideran como conceptos distintos. Así: "el multilingüismo es el conocimiento de varias lenguas o la coexistencia de varias lenguas en una sociedad determinada", enfatizando que "se puede lograr simplemente diversificando las lenguas que se ofrecen en un centro escolar o en un sistema educativo concretos, procurando que los alumnos aprendan más de un idioma extranjero" (Ministerio de Educación, Cultura y Deporte, MECD, 2002, p.4). En el Marco Europeo, se defiende que el objetivo es que las personas puedan interactuar y aprovechar todos los recursos de las lenguas que conocen, aunque no las dominen, para comunicarse. No se trata, por tanto, de dominar las lenguas como un nativo, sino de "tener un repertorio lingüístico en el que tengan lugar todas las capacidades lingüísticas" (MECD, p. 4), esto es, se relaciona con la interrelación entre las competencias en varias lenguas (en ello consiste la competencia plurilingüe) $)^{1}$.

Más allá de esta complejidad terminológica, la clave para la comprensión del fenómeno del bilingüismo está en las propias variables que impiden un acuerdo claro sobre su definición y que son esenciales para valorar cada caso. Entre ellas destacan: el momento de aprendizaje de cada lengua, las demandas del entorno, el tipo de exposición (inmersión vs. formal; familiar vs. no familiar), la composición familiar, el tiempo de exposición, la cantidad de exposición, la competencia lingüística, pragmática y/o sociolingüística, y la competencia oral, escrita y/o académica (Genesee, 2006; Hoff y Core, 2013).

La influencia de algunas de estas variables puede valorarse en sociedades occidentalizadas, no así en muchas zonas del mundo donde la lectura y los medios de información no están al alcance de todos. No obstante, ello no impide el desarrollo del

\footnotetext{
${ }^{1}$ Con el fin de clarificar la información, a partir de aquí nos referiremos con el término bilingüismo a situaciones que impliquen dos o más lenguas, orales o signadas. En las ocasiones donde los resultados de estudios referidos hagan referencia situaciones de multilingüismo, se especificará.
} 
bilingüismo. Esto conduce a que la definición que se utiliza en cada investigación pueda variar en función de variables culturales y/o por la orientación teórica de los investigadores y las preguntas concretas que se plantean. La mayor parte de las investigaciones disponibles no muestran en detalle cuáles son los criterios de inclusión de participantes de los que se han servido, lo que dificulta la comparación de resultados y por consiguiente, el avance en la investigación.

Ante esta variedad de enfoques, puede verse que las definiciones generales no resultan muy útiles a nivel clínico. El logopeda debe conocer y comprender estas variables que pueden influir en el aprendizaje de dos o más lenguas. La variación, por ejemplo, en el tiempo de exposición, puede traducirse en variaciones de procesamiento, ya que las diferencias entre bilingües y monolingües son más o menos amplias en los distintos momentos del desarrollo, lo que convierte esta variable en un criterio más recomendable que la edad cronológica (Goldberg, Paradis y Crago, 2008). Por otro lado, debido a la complejidad del fenómeno y a la enorme variabilidad individual que se encuentra en su estudio, el bilingüismo debería entenderse como un continuo. En un extremo de este continuo se situaría el bilingüismo simultáneo o familiar (aprendizaje de las lenguas desde la infancia), seguido de las diferentes situaciones de aprendizaje de una segunda lengua (relacionadas con los distintos modelos de escolarización o cambios de domicilio, entre otros factores) y en el otro extremo se encontrarían los casos de bilingüismo sustractivo o diglosia (donde una de las lenguas de un sujeto potencialmente bilingüe está en una situación de desequilibrio que impide su desarrollo).

Para poder valorar el grado de bilingüismo en este continuo, es necesario tener en cuenta tanto la competencia gramatical como funcional del usuario dentro de las limitaciones sociales y comunicativas de la persona. En consecuencia, sería más apropiado referirse a varios tipos de bilingüismo, dependiendo de las situaciones (o situación de la lengua) y el tipo de competencia y procesos de aprendizaje de las personas bilingües, que a bilingüismo como término genérico. Las situaciones vienen determinadas por el estatus de la lengua y por el uso que se hace de ella. La lengua puede estar en una situación de equilibrio o en una situación donde exista una lengua mayoritaria y una lengua minoritaria en función del estatus social o político y de la predominancia de uso por la población autóctona, entre otros factores.

Esta situación cuando es de desequilibrio puede provocar un entorno que favorezca el bilingüismo aditivo, donde ambas lenguas se enriquecen culturalmente al 
estar en contacto, o por el contrario promover un bilingüismo sustractivo, en el que una de las dos lenguas se desarrolla en detrimento de la otra. En ocasiones, el bilingüismo sustractivo puede desembocar a nivel social en situaciones de diglosia, en las que una de las lenguas se considera de prestigio y ostenta privilegios sociales o políticos, y a nivel de la persona, en un alejamiento de una de las lenguas, pudiendo fomentar su olvido y/o o en dificultades de identificación (bilingüismo acultural) con el consecuente rechazo de la cultura relacionada (Lambert, 1974)

A lo largo de este artículo utilizaremos la nomenclatura habitual para hablar de las diferentes lenguas que utiliza una persona: refiriéndonos a la primera lengua o lengua dominante del usuario en relación a la lengua que mejor domina en un momento concreto, no siendo esta necesariamente la primera que adquirió. En el caso de los niños, suele corresponder con lengua materna hablada en casa. La segunda lengua o lengua no dominante será la otra lengua. En cuanto al aprendizaje de esta segunda lengua, existen contextos de bilingüismo simultáneo, cuando las dos lenguas se aprenden antes de los tres años, y de bilingüismo sucesivo, en los que la segunda lengua se aprende cuando el usuario ya puede comunicarse de forma básica en la primera lengua. Habitualmente, el bilingüismo sucesivo está relacionado con la escolarización en una lengua distinta de la del hogar o con una escolarización bilingüe.

La diferente temporización en la exposición a las lenguas del bilingüe afecta a los procesos de aprendizaje y ha de tenerse en cuenta en el planteamiento de la evaluación e intervención logopédica, sea cual sea la lengua en la que se intervenga (Hammer et al. 2014).

A su vez, la adquisición más o menos temprana de una lengua puede relacionarse con el nivel de expectativas de los usuarios y familiares, así como con otras variables emocionales que indirectamente influirán en el éxito de la terapia (De Houwer, 1999a; Räsänen y Pine, 2014).

\section{Atención educativa en entornos multilingües y multiculturales}

En el Estado Español, las distintas situaciones bilingües que se encuentran pueden estar asociadas a diferentes zonas geográficas y/o a movimientos migratorios. Los logopedas deben conocer cuáles son los modelos de atención educativa vigentes, cuyas competencias vienen reguladas por las diferentes autonomías. Se encuentran tres 
contextos educativos relacionados con población bilingüe o potencialmente bilingüe ${ }^{2}: 1$ ) Educación bilingüe en lenguas extranjeras; 2) Educación bilingüe en lenguas cooficiales o en dialectos regionales; 3) Educación bilingüe en lenguas de signos y lenguas orales.

\section{Educación bilingüe en lenguas extranjeras}

En el contexto de una educación en lenguas extranjeras, una de las lenguas es la lengua mayoritaria del territorio donde se estudia y la otra una lengua no oficial no hablada mayoritariamente en ese territorio. Generalmente la lengua no oficial es el inglés, en algunos casos el francés o el alemán. Se trata de colegios públicos, concertados o privados con programa bilingüe (habitualmente con una dedicación a partir de un 30$40 \%$ del horario lectivo), con programa educativo español o combinado. En las zonas con lenguas cooficiales, el porcentaje de dedicación a cada lengua se divide entre el castellano y la lengua de la zona.

Dentro de este sistema, existen también colegios privados cuyos programas están regulados por otros países, como es el caso de los colegios alemanes, liceo francés o colegios británicos, donde el modelo es de inmersión durante las horas lectivas. Es decir, la escolarización, salvo la/s asignatura/s de lengua/s del territorio (lengua española, catalán, euskera, etc.), son las del plan de estudios del país extranjero y se imparten en su lengua, además de utilizarse la lengua extranjera para la interacción no formal dentro del centro de estudios.

\section{Educación bilingüe en lenguas cooficiales o en dialectos regionales}

En los modelos de educación en lenguas cooficiales o en dialectos regionales existen diferentes formas de implantación del bilingüismo, desde los modelos en los que predomina la lengua cooficial respectiva en la enseñanza, y el castellano y las lenguas extranjeras se imparten como materia, a modelos predominantemente bilingües; pudiendo llegar a situaciones como la del Valle de Arán (Cataluña), donde los niños aprenden progresivamente aranés, catalán, castellano, inglés y francés (González i Planas, 2002).

\footnotetext{
${ }^{2}$ Es necesario puntualizar que los casos de adopción internacional no se han considerado dentro de estos perfiles, ya que los niños adoptados solo son bilingües durante un corto período de tiempo. Al no hablar los padres adoptivos su lengua de origen, estos niños pierden generalmente sus habilidades expresivas aproximadamente al año de ser adoptados (Gindis, 1999).
} 
La implantación de uno u otro tipo de modelo varía con el tiempo en las diferentes regiones y depende de decisiones institucionales.

\section{Educación bilingüe en lenguas de signos y lenguas orales}

Potencialmente la Educación bilinguie en lenguas de signos y lenguas orales es una situación asimilable a la educación en lenguas cooficiales, no obstante, por su particularidad, trataremos este contexto en un apartado distinto.

Según la legislación (Ley 27/2007, de 24 de octubre) "Las Administraciones educativas ofertarán, en los centros que se determinen, entre otros, modelos educativos bilingües, que serán de libre elección por el alumnado sordo, con discapacidad auditiva y sordociega o sus padres o representantes legales, en el caso de ser menores de edad o estar incapacitado”. En la práctica, no hay aplicación de esta ley. Si se utiliza la lengua de signos se considera un colegio como bilingüe, aunque la exposición sea limitada o una herramienta de apoyo.

No obstante, se han encontrado resultados positivos de la exposición bilingüe a la lengua oral y signada en niños sordos. En un estudio reciente con metodología longitudinal llevado a cabo por Pérez Martín, Valmaseda Balanzategui y Morgan (2014) a partir de una muestra de sordos y oyentes con un modelo de bilingüismo simultáneo en lengua oral y en lengua de signos, se observó un progreso en ambas lenguas en los dos grupos, con habilidades lingüísticas dentro de su edad cronológica y auditiva. Estos resultados van en la misma dirección de investigaciones anteriores en otras lenguas, como el inglés y la Lengua de Signos Americana (Pettito et al. 2001).

\section{Atención a la diversidad multicultural}

Además de estos modelos de educación bilingüe, existe otro contexto educativo relacionado con población potencialmente bilingüe. Se trata de los programas de integración lingüística que tratan de atender a la diversidad multicultural y multilingüística de las poblaciones de origen inmigrante. Hasta ahora se han enunciado propuestas específicas (Morales Puertas, 2000), y desarrollado contextos en los que llevarlas a cabo, como es el caso de las Aulas de Enlace ${ }^{3}$. Estas aulas se encuentran

\footnotetext{
${ }^{3}$ Adoptamos la denominación de la Comunidad de Madrid, aunque existen otras denominaciones en las diferentes comunidades autónomas: Aulas Temporales de Adaptación Lingüística (ATAL) en Andalucía y Extremadura, Plan de Acogida del Centro Docente en Cataluña, Islas Baleares y Murcia, Aulas Temporales de Inmersión Lingüística (ATIL) en Asturias, Aulas de Inmersión Lingüística (AIL) en Navarra y La Rioja, Aulas de Adaptación Lingüística y Social (Aulas ALISO) en Castilla-León, Grupos
} 
dentro de los centros educativos. Su objetivo es incorporar a los hijos de inmigrantes al sistema educativo mediante la enseñanza de la lengua de la comunidad de acogida, en algunos casos por falta de dominio de la lengua vehicular y en otros para mejorar la adaptación al currículo. Según un estudio de Fernández, Delgado, Herrero y Permisán (2010), realizado con población de origen inmigrante, el modelo de las Aulas de Enlace tiene como consecuencia un bilingüismo sustractivo, ya que el objetivo es asimilar la cultura de origen a la cultura y lengua de acogida y los profesores a cargo de estas aulas no suelen conocer las lenguas maternas de los alumnos.

Martín Rojo y Mijares (2007, p.1) analizan dos posibilidades en el enfoque de la diversidad cultural: "la pluralidad lingüística entendida como un obstáculo o aprovechada para el aprendizaje" y observan que existe una creencia extendida de que las lenguas de los inmigrantes no favorecen la integración. Fernández et al. (2010), resaltan que no es la única visión posible, ya que en otros países se tiende a un bilingüismo aditivo.

En varias zonas del Estado Español, la situación de bilingüismo se vuelve más compleja, al mezclarse los programas de inmersión lingüística de las lenguas cooficiales con la enseñanza de las lenguas extranjeras y/o con la atención a la diversidad de origen inmigrante, donde gran número de niños tienen una lengua de partida normalmente diferente de la/s de acogida (Huguet et al. 2006; Huguet, Chireac, Navarro y Sansó, 2011). En España, existen datos de escuelas trilingües en el País Vasco (668), Cataluña (20) y la Comunidad Valenciana (161). La introducción de una tercera lengua en la Comunidad Valenciana se realiza a los 3 ó 6 años, en el País Vaco a los 4 años y en Cataluña a los 3 ó 6 años (Riemersma y De Vries, 2011). Desde la Unión Europea se recomienda aumentar la investigación sobre estas prácticas, fijar las políticas de apoyo para la formación de profesorado y facilitar una mayor accesibilidad a materiales educativos.

\section{Resultados preliminares de los programas de educación bilingüe}

Como logopeda, es fundamental tener una visión crítica de los programas de educación bilingüe, porque su disponibilidad no siempre es garantía de éxito. Es decir, no todos los niños escolarizados en programas bilingües llegarán a desarrollar una buena 
competencia en todas las lenguas, ya que su implantación puede ser en ocasiones inadecuada. Sin embargo, al no contar aún con datos fiables sobre las diferencias en las competencias lingüísticas entre alumnos de escuelas bilingües y alumnos de programas no bilingües, se debe interpretar la información disponible en los medios de comunicación y en fuentes científicas con precaución, dado que la complejidad de las diferentes situaciones linguiísticas en cada autonomía y de las situaciones personales de cada individuo bilingüe dificultan una evaluación general de los resultados.

Concretamente, los programas de educación bilingüe en lenguas extranjeras están aún en proceso de implantación y presentan algunas dificultades, como la selección del profesorado. Según la Ley Orgánica de Mejora de la Calidad Educativa (LOMCE 8/2013, de 9 de diciembre): los profesores serán «Expertos con dominio de lenguas extranjeras. Para cada curso escolar, las Administraciones educativas podrán excepcionalmente, mientras exista insuficiencia de personal docente con competencias lingüísticas suficientes, incorporar expertos con dominio de lenguas extranjeras, nacionales o extranjeros, como profesorado en programas bilingües o plurilingües". Fernández y Halbach (2011) destacan que a los profesores se les exige competencia lingüística, no necesariamente metodológica, y se les da una formación de tres meses para adaptarse mediante el programa de Aprendizaje Integrado de Contenidos y Lengua Extranjera, AICLE (Fernández Fontecha, 2001). Los programas de formación no mencionan la necesidad de un entrenamiento específico de base pedagógica para la enseñanza bilingüe.

Las carencias en la formación del profesorado pueden repercutir en el éxito de esos programas. La evaluación de los resultados a largo plazo permitirá valorar la repercusión de su implementación en el aprendizaje.

Sin embargo, los programas bilingües en lenguas cooficiales, llevan más tiempo de implantación y los resultados parecen ser más positivos, lo que indica que el hecho de que la lengua sea una lengua vehicular en el lugar donde se estudia pueda influir favorablemente. Sin embargo, para poder valorarlo es necesario hacer análisis que comparen los diferentes grados de inmersión y tiempo que se le dedica a cada lengua en la escuela y en los contextos familiar y social (Huguet, 2004).

\section{Bilingüismo y desarrollo del lenguaje}

Los diferentes estudios sobre desarrollo del lenguaje y bilingüismo tienen un objetivo común: la comprensión de las características y variedad del input lingüístico para los 
bilingües y su influencia en el desarrollo (Hoff y Core, 2013). Concretamente se han centrado en los siguientes temas: percepción del habla, descripción de perfiles y trayectorias de desarrollo, separación de las lenguas en uno o varios sistemas, relación entre lengua y nivel de lectoescritura, transferencia de los conocimientos aprendidos de una lengua a otra, influencia del entorno, y evaluación e intervención en alteraciones del habla y el lenguaje (véase Hammer et al. 2014, para una revisión).

Existen varias creencias sobre el aprendizaje bilingüe que han dificultado la valoración positiva de los entornos educativos bilingües durante gran parte del siglo $\mathrm{XX}$ (Werker y Bryers-Heinlein, 2008). En algunos países o zonas, el bilingüismo puede ser una elección, sin embargo, en otros lugares es una realidad social a la que es necesario dar respuesta. Posiblemente por la dificultad que entraña para los adultos el aprendizaje de una segunda lengua, ha arraigado la idea de que aprender dos lenguas es una carga cognitiva adicional y puede confundir a los niños. En relación con esta idea, existe la percepción de que un bilingüe es "la suma de dos monolingües". Intuitivamente parecería que si los bilingües pasan menos tiempo en contacto con cada una de sus lenguas del que pasa un monolingüe, no podrían aprenderlas del mismo modo. Este concepto ha promovido la filosofía extendida de la fórmula de Grammont una persona una lengua, referida a la separación entre las lenguas con las que interaccionan los interlocutores con el niño, permitiendo asociar cada lengua a una persona concreta que sólo se dirige al niño en esa lengua. Por ejemplo, si los padres tienen dos lenguas maternas distintas, cada padre hablaría al niño en su lengua. Así, un padre podría utilizar siempre el castellano para dirigirse al niño y la madre el francés. La aceptación de esta fórmula se traduce o en la recomendación de separar las dos lenguas disponibles para evitar un esfuerzo extra en el aprendizaje o confusión (Ronjat, Escudé y Lieutard, 1913, citado por Barron-Hauwaert, 2004). Hoy en día los estudios discrepan sobre la necesidad y eficacia de esta estrategia (Conboy, Brooks, Meltzof y Khul, 2015; de Houwer, 2009; Döpke 1998; Yamamoto, 2002).

La realidad muestra una gran heterogeneidad en el desarrollo del lenguaje, incluso en contextos monolingües de aprendizaje. Aprender dos lenguas podría ser un proceso más lento que aprender una a nivel de vocabulario y de desarrollo gramatical (Hoff y Core, 2013).

Existen varios factores que pueden afectar a este ritmo de aprendizaje y que podrían ser buenos predictores del desarrollo partiendo de las características específicas del desarrollo bilingüe. Entre ellos se encuentran las características en el input que 
afectan a la cantidad y la calidad de la exposición. Estas características están relacionadas con la composición familiar, la calidad del input y la competencia lingüística de los padres (el grado de dominio de su lengua a nivel formal y funcional).

A continuación se describen varios de estos predictores con un resumen de los resultados de distintas investigaciones.

\section{Variables relativas a las medidas de producción lingüística}

Los aspectos lingüísticos que más se han estudiado han sido el desarrollo del vocabulario y de la competencia gramatical. Este desarrollo, en ocasiones más lento, en los niños bilingües, es una preocupación que comparten muchos padres.

Se encuentran resultados contradictorios en relación al desarrollo del vocabulario, posiblemente debido a que los niños bilingües son a menudo evaluados en sus dos lenguas por separado, como se haría con dos personas monolingües de cada lengua, y comparados con los monolingües con test baremados para estas poblaciones. Teuber y Furlong (1985) obtuvieron resultados en vocabulario para bilingües de dos desviaciones típicas por debajo de los monolingües. Pearson, Fernández y Oller (1993) encontraron la misma producción de vocabulario en bilingües y monolingües a partir de los resultados del Inventario de Desarrollo Comunicativo MacArthur (Fenson et al. 1993). Su estudio tuvo en cuenta el vocabulario conceptual global (en el que los términos usados en ambas lenguas, los equivalentes de traducción, contabilizan como un único término). Águila, Ramón-Casas, Pons y Bosch (2007) obtuvieron resultados que situaban a los bilingües por encima de los monolingües al combinar su vocabulario.

La discrepancia en los resultados de estas y otras investigaciones pueden deberse, por tanto, a diferencias metodológicas. Para comprender estas diferencias, es importante reflexionar sobre las implicaciones del uso de cada criterio de medida de vocabulario. Así, el estudio de Hoff, et al. (2012) ilustra cómo pueden obtenerse resultados diferentes. Estas autoras realizan una comparación entre los resultados de dos medidas: una medida estándar de vocabulario que consiste en medir la producción de palabras en cada lengua del bilingüe por separado, y una medida de vocabulario total (suma global de todas las palabras en ambas lenguas considerándolas como conjunto único, incluyendo los equivalentes de traducción en las dos lenguas.) Por ejemplo, si un niño tiene en su repertorio de inglés y castellano las palabras puppet y muñeco se contabilizan como dos palabras, ya que los contextos de uso y el tipo de objeto no son exactamente equivalentes, y se suman todas las palabras producidas en los dos idiomas. 
El estudio se basa en el análisis de una muestra de niños del mismo nivel socioeconómico residentes en Florida divididos en dos grupos: 1) monolingües de inglés y 2) bilingües de inglés y español (siendo la proporción media de exposición al inglés en este grupo del 51\%). El análisis de la medida estándar de vocabulario mostró niveles más bajos de vocabulario en los bilingües que en los monolingües de inglés. Sin embargo, al combinar ambas lenguas en una media de vocabulario total no encontraron diferencias entre bilingües y monolingües.

El vocabulario total se ha utilizado en estos estudios al entender que los equivalentes de traducción no tienen por qué ser una traducción exacta de un mismo concepto. Los niños suelen tener un vocabulario diferente en ambas lenguas porque sus experiencias son distintas. La inestabilidad y variación en el vocabulario temprano no permite establecer relaciones inequívocas entre ambos vocabularios. Es por esto que el vocabulario total podría resultar una medida más fiable que el vocabulario conceptual. Atender a estas diferencias es crucial para comprender los resultados de las investigaciones y para planificar una buena evaluación del lenguaje en niños bilingües.

Un estudio reciente más completo sobre niños hablantes de holandés y francés apoya los anteriores resultados para otras lenguas (De Houwer, Bornstein y Putnick, 2014). En este trabajo, el $71 \%$ de los bilingües tenían niveles más altos de comprensión, al combinar ambas lenguas. No encuentran, por tanto, diferencias entre bilingües y monolingües, concluyendo que la exposición a dos lenguas "no ralentiza el desarrollo léxico" (De Houwer et al., 2014, p.21). Estas autoras resaltan la importancia de partir de una muestra homogénea, especialmente en el aspecto socioeconómico, para la fiabilidad de los estudios. Aún así, la enorme variabilidad de circunstancias o contextos bilingües dificulta la comparación de resultados.

En esta línea, Hoff y Core (2013) observaron que la lengua dominante en casa coincide con la dominante en vocabulario y con la lengua en la que se inicia la combinación de palabras en edades tempranas (en bilingües inglés español de 22 y 30 meses, con medidas del MacArthur). En cuanto al desarrollo gramatical, estas investigadoras encontraron que los niños monolingües de inglés presentaban medidas más avanzadas en Longitud Media de Emisión y complejidad gramatical.

En cuanto a los patrones de desarrollo, existe un acuerdo en que los bilingües muestran un ritmo de desarrollo morfosintáctico equivalente al de los monolingües en su lengua dominante (De Houwer, Bornstein y Leach, 2005). No obstante, Thordardottir, Rothenberg, Rivard y Naves (2006) sugieren un desfase temporal entre 
los niños bilingües y monolingües en inicio de la combinación de palabras. Hay evidencia de que este desfase puede desaparecer si se acepta como logro su inicio en cualquiera de las lenguas del bilingüe (Hoff et al., 2012).

Si nos centramos en el desarrollo fonológico, tradicionalmente ha habido un debate sobre si los bilingües poseen dos o un único sistema fonológico (Paradis, 2001). Actualmente se considera que el sistema fonológico de los bilingües consiste en dos subsistemas que interaccionan entre sí. Hasta ahora las investigaciones se han centrado en los factores que intervienen en las interacciones, más que en examinar el modo en que interaccionan (Hambly et al. 2013). La interrelación entre ambos sistemas puede ser positiva o negativa, es decir, que los aprendizajes en una lengua favorezcan o dificulten el avance en otra (Goldstein y McLeod, 2012). En Hambly et al. (2013) puede consultarse una revisión de trabajos de los últimos 50 años, divididos en estudios sobre percepción, producción y procesamiento fonológico. Apoyándose en resultados de investigaciones como las de Yavas y Goldstein (2006), afirman que "no existe una clara evidencia que sugiera que los niños bilingües desarrollen el habla a un ritmo más lento que sus compañeros; sin embargo, sí hay evidencias cualitativas" (Hambly et al. 2013, p.12). No obstante, los niños bilingües pueden adquirir antes o más tarde algunos fonemas, dependiendo de las interacciones entre lenguas y estructuras. Para estudiar estos aspectos, recomiendan realizar estudios longitudinales y la comparación entre bilingües simultáneos y sucesivos. Se han encontrado diferencias en percepción temprana y distintos patrones de desarrollo fonológico (Genesee y Nicoladis, 2007). Del mismo modo, algunos autores afirman que los bilingües con desarrollo típico muestran una inteligibilidad más baja que los monolingües, con más sonidos distorsionados, patrones de interferencia entre lenguas y patrones de error atípicos (Iglesias y Goldstein, 1998). Estos errores poco comunes no tienen por qué ser un indicador de patología en niños bilingües (Hambly, Wren, McLeod y Roulstone, 2013). Los patrones de error típicos en una lengua pueden no serlo en otra.

Según Yavas y Goldstein (1998), existen patrones de error específicamente bilingües: la no diferenciación entre sonidos que en una lengua son alófonos y en otra no, o a la inversa, la sustitución de unos fonemas por otros en su otra lengua, el uso de un ritmo no predominante en una lengua (p.ej. un niño bilingüe español-francés podría usar un ritmo yámbico ${ }^{4}$ típicamente francés para palabras en español, cuando el ritmo

\footnotetext{
${ }^{4}$ Distribución temporal de acentos y pausas de las palabras con tonicidad en la última sílaba, el resto átonas.
} 
predominante en esta lengua es el trocaico ${ }^{5}$ ), los errores en la duración y en el tono de las sílabas. Lógicamente, para poder valorar estos patrones es necesario conocer la variedad dialectal a la que está expuesto el niño.

No obstante, las diferencias que se observan en los estudios anteriores no parecen ser permanentes. Así, Gathercole y Thomas (2009) encuentran que el desfase entre monolingües y bilingües puede recuperarse a los 9 ó 10 años de edad.

Por otra parte, es necesario prestar atención a otros aspectos de la comunicación más allá de la producción oral. El creciente interés por los estudios multimodales ha llevado a los investigadores a estudiar la producción gestual de los bilingües, informando de un mayor uso de gestos manuales que en monolingües (Nicoladis, Pika y Marentette, 2009), con diferencias en función de la cultura (Smithson, Nicoladis y Marentette, 2011).

Otro campo de estudio es la transferencia de los aprendizajes entre lenguas. En los trabajos sobre lectoescritura se ha visto que tanto los prerrequisitos lectores como las habilidades de decodificación pueden ser transferibles de una lengua a otra (August y Shanahan, 2006). Es decir, los niños que aprenden a decodificar los grafemas de una de sus lenguas, pueden usar estas habilidades para decodificar en su otra lengua.

\section{Variables relativas al entorno lingüístico}

En la investigación sobre bilingüismo no existe un corpus de estudio suficiente aún que permita predecir los resultados de la condición bilingüe de un niño. Se insiste en que la exposición y el entorno tienen un papel importante, ya que educarse en un ambiente bilingüe no garantiza que los niños vayan a ser bilingües con el paso del tiempo. Si la exposición no es suficiente, podrían dejar de usar una de sus lenguas en un futuro (Pearson y Fernández, 1994).

Algunos investigadores han mostrado cómo un umbral mínimo de exposición del 20\% del input total a una lengua puede ser suficiente (Hoff et al. 2012). Sin embargo, el umbral podría ser distinto para la comprensión.

Varios estudios han permitido destacar como buen predictor del éxito en el desarrollo de ambas lenguas, de que el niño sea finalmente bilingüe, que los dos padres hablen la lengua minoritaria y que sean nativos (De Houwer, 2007; Hakutaka y D’Andrea 1992).

\footnotetext{
${ }^{5}$ Distribución temporal de acentos y pausas de las palabras con tonicidad en la penúltima sílaba de la palabra, el resto átonas.
} 
Por otra parte, las propiedades del habla de las personas con las que el niño interacciona en su entorno (input) también son buenas predictoras: el uso de vocabulario variado y de estructuras sintácticas variadas por parte de las personas con las que interacciona. En el caso, por ejemplo, de que el niño interactúe con personas distintas, le permite estar expuesto a una mayor variedad fonológica y de formas sintácticas y léxicas (Place y Hoff, 2011). También han resultado buenos predictores del desarrollo bilingüe el uso de un lenguaje descontextualizado (hablar sobre situaciones y personas no presentes en el momento de la interacción) y la lectura de cuentos (Hoff y Core, 2013).

Existen diferentes metodologías, algunas más populares que otras, para sistematizar el uso de las lenguas en el entorno del niño: 1) una persona una lengua, relacionada con la separación de lenguas por interlocutores), 2) tiempo y lugar, donde las dos lenguas no se separan por el interlocutor, sino por lugares de uso. Por ejemplo, los fines de semana o en casa de los abuelos se cambia la lengua hablada en la familia. Esta estrategia puede incluir otra: la lengua minoritaria en casa, para potenciar este tipo de lenguas. Finalmente, 3) la estrategia mixta, en la que los padres intercalan las dos lenguas en una misma conversación (Pearson, 2010). En relación con la mezcla de lenguas en el input, no está claro su efecto, salvo si la mezcla es en una misma frase. Place y Hoff (2010) encuentran una correlación negativa de estas prácticas en una misma frase con la comprensión temprana de vocabulario.

Los resultados de las investigaciones disponibles destacan que la estrategia lengua minoritaria en casa produce el bilingüismo más activo. Asimismo, defienden la importancia de observar la lengua en la que hablan los padres entre sí, ya que se han obtenido mejores resultados cuando los dos padres hablaban la lengua minoritaria (De Houwer, 2009).

\section{¿Diferencias o dificultades?}

A pesar de estos predictores, existe un conflicto en la práctica para diferenciar las particularidades del desarrollo bilingüe de las dificultades que pueden desembocar en patología del lenguaje. Sin olvidar que aún se está discutiendo sobre los criterios de identificación de los hablantes tardíos en niños monolingües (Rescorla y Dale, 2013).

Pearson (2013) propone varios principios a tener en cuenta en la evaluación de niños bilingües para diferenciarlos de los hablantes tardíos, entre los que destacan los siguientes: 1) distinguir si las medidas obtenidas son más bajas o se trata de un desfase 
temporal representativo en el inicio de una habilidad. Si es el segundo caso, puede ser una dificultad, tanto en bilingües como en monolingües. Un ejemplo es la ausencia de combinación de palabras a los 30 meses, en enunciados de cualquier lengua aislada o en enunciados en los que se mezclan ambas lenguas; y 2) evitar generalizar los logros aislados como habilidades productivas. La relación entre variables no tiene que ser necesariamente la misma para los bilingües.

El avance de la investigación en el campo de la adquisición del lenguaje infantil permitirá una mayor comprensión de las distintas trayectorias posibles para poder distinguir los casos que presentan dificultades en el aprendizaje de los casos en los que las discrepancias se deben a diferencias individuales.

Gran parte de los estudios recientes sobre desarrollo lingüístico en niños bilingües se ha centrado en el Trastorno Específico del Lenguaje (TEL). Existe evidencia que indica que sus dificultades se manifiestan en todas sus lenguas (Ebert, Kohnert, Pham, Disher, y Payesteh, 2014) y que los errores que cometen son similares a los de los niños monolingües con TEL (Jacobson y Schwartz, 2005), aunque también hay estudios que lo contradicen, encontrando resultados inferiores para los bilingües (Orgassa y Weerman, 2008). Estas dificultades podrían ser de dominio general, es decir, relacionadas con el procesamiento cognitivo, no necesariamente lingüístico (véase Miller, 2011 para una revisión).

Se ha observado que los niños bilingües con TEL muestran resultados equivalentes a los de los niños monolingües con TEL, tanto en tareas de procesamiento cognitivo (Kohnert, Windsor y Ebert, 2009), como en competencia morfosintáctica (Paradis, 2007). En cuanto a los procesos lectores, se encuentran trabajos que registran cómo los déficits en procesamiento fonológico (conciencia fonológica, memoria fonológica y denominación automática rápida), atención (auditiva y visual) y procesamiento del lenguaje (comprensión de instrucciones, repetición de frases y fluencia verbal), al inicio del contacto con la lectura pueden estar relacionados con dificultades posteriores en lectoescritura (Aguilar Mediavilla et al. 2014).

Estos resultados sugieren que es importante comparar a los niños bilingües con TEL con los bilingües de desarrollo típico y/o con los monolingües con TEL, por las particularidades que presentan los bilingües en cuanto al procesamiento. 


\section{Beneficios cognitivos y sociales del bilingüismo}

Cada vez disponemos de una mayor evidencia empírica en defensa de los beneficios de ser bilingüe y este es un tema de interés actual, tanto en los medios, como en el ámbito de la investigación (Bialystok, 2015). Se recomienda interpretar con precaución la información disponible ya que no todos los estudios encuentran ventajas cognitivas a favor de los bilingües (Costa, Hernández, Costa-Faidella y Sebastián-Gallés, 2009).

Entre los beneficios que se defienden, se encuentran los directamente relacionados con la lengua y los sociolingüísticos y emocionales. Los niños bilingües tienen conciencia de la propiedad de arbitrariedad del lenguaje desde muy temprano, una mayor conciencia fonémica (ya que las lenguas pueden tener inventarios fonológicos distintos), mayor flexibilidad y apertura a otras lenguas y culturas (entendiendo que su perspectiva es una entre otras posibles), beneficios de adaptación a la comunidad y mejores opciones de trabajo y movilidad (Pearson, 2010).

Esta flexibilidad y apertura puede observarse en los trabajos de Wright y Tropp (2005) sobre las preferencias de 351 niños norteamericanos blancos caucásicos de entre 5 y 9 años por conocer amigos latinos. Los niños americanos estaban escolarizados en tres grupos: 1) predominantemente blancos con enseñanza monolingüe en inglés, 2) mezcla étnica ${ }^{6}$ con enseñanza monolingüe en inglés y 3) mezcla étnica con enseñanza bilingüe inglés-español. Ante la presentación de fotos de niños latinos y blancos, se les pedía que eligieran qué niños podrían ser sus amigos. Hallaron una mayor preferencia por los niños latinos entre los niños blancos escolarizados en modelo bilingüe (un $40 \%$ frente al $10 \%$ en los escolarizados en inglés sin mezcla étnica y al 12-13\% en los escolarizados en inglés con mezcla étnica), por lo que compartir la lengua puede verse como un factor más allá de la tolerancia racial por contacto.

En cuanto a los beneficios cognitivos de ser bilingüe, uno de los hallazgos principales es el relativo a las habilidades de dominio general, concretamente las que se relacionan con las funciones ejecutivas, que tienen consecuencias no solo en el procesamiento lingüístico en ambas lenguas, sino en el procesamiento de tareas no lingüísticas. Los bilingües muestran una mejora del control ejecutivo como resultado del manejo de sus dos lenguas, activas en todo momento, aun en contextos de uso monolingüe: ya que tienen que seleccionar constantemente una lengua cuando ambas compiten e inhibir el uso de la/s otra/s. Ellen Bialystok (véase Biakystok, 2011, para

\footnotetext{
| ${ }^{6}$ Escolarización en una misma clase de niños norteamericanos caucásicos y niños latinos.
} 
una revisión) ha centrado su investigación en el estudio experimental de estas funciones en tareas de control inhibitorio, cambio de tarea, resolución de tareas con conflicto, y de tareas que requieren inhibir una respuesta habitual. Un ejemplo de este tipo de tareas es la tarea de Stroop (Stroop, 1935, citado en MacLeod, 1991).

En bilingües de lengua de signos también se encuentran estos efectos, no así en niños expuestos al lenguaje bimodal, por lo que no parecen deberse al canal visogestual, sino a la condición bilingüe, como se observa en un estudio de Emmorey, Luk, Pyers, y Bialystok (2008) en el que los niños expuestos al bimodal que realizaron tareas de reconocimiento de estímulos congruentes e incongruentes (flanker task), obtuvieron resultados similares a los monolingües.

A pesar de que el control ejecutivo decrece con la edad (Daniels, Toth y Jacoby, 2006), la ventaja de los bilingües en este mecanismo con respecto a los hablantes monolingües se mantiene a lo largo del ciclo vital y mejora con la práctica. Esto puede verse a través de la comparación de resultados clínicos de usuarios con diagnóstico de demencia. En una muestra de 91 usuarios monolingües y 93 bilingües, la mayor parte con Alzheimer, Bialystok, Craik y Freedman (2007) hallaron un desfase significativo en la edad en la que empiezan a mostrar síntomas (una variación de 4.1 años) a favor de los bilingües. Más tarde confirmaron que los altos niveles de desempeño de los bilingües en tareas que implican funciones ejecutivas, tanto en tareas verbales como no verbales, están por encima de su nivel educativo (Craik, Bialystok y Freedman, 2010) incluso cuando la enfermedad está más avanzada a nivel anatómico-funcional en bilingües (Frisoni, Rossi y Beltramello, 2002). Se defiende la hipótesis de que los bilingües son capaces de compensar mediante su reserva cognitiva, creando una red “más difusa, bilateral y eficiente" (Bialystok, 2011, p.233).

Otra ventaja en el procesamiento de la información por parte de los bilingües tiene que ver con las habilidades metalingüísticas, que se incrementan con el tiempo de exposición a la lengua. En un estudio de Bialystok, Peets y Moreno (2014) en el que comparan los conocimientos metalingüísticos de un grupo de monolingües de inglés con un grupo de bilingües en un programa de inmersión en francés, obtienen mejores resultados en el grupo bilingüe.

Los futuros avances en la investigación permitirán matizar estos resultados y comprobarlos en distintas poblaciones para que puedan ser de mayor utilidad para la terapia del lenguaje en los niños bilingües y en los adultos con dificultades. 


\section{Orientaciones para una evaluación e intervención basada en la evidencia}

En el caso de las alteraciones del lenguaje, el desconocimiento de las particularidades de los niños bilingües complica la toma de decisiones sobre qué son diferencias debidas a procesos propios del desarrollo bilingüe y qué son dificultades. Gran parte de los logopedas orientan a los padres a utilizar sólo una lengua con niños que presentan alteraciones. Esta práctica no siempre está basada en la evidencia científica. Además, en algunos contextos no es posible sin limitar las oportunidades de comunicación del niño en su entorno y la construcción de su identidad cultural. Sin una valoración de cuál es la secuencia de aprendizaje de las lenguas del usuario y su lengua dominante la intervención puede fracasar. Tal es el caso de niños escolarizados en una lengua mayoritaria en cuyas casas se habla una lengua minoritaria, o de los niños que crecen en regiones donde coexisten varias lenguas de uso común.

\section{Logopedia y atención a la diversidad cultural}

Ante los crecientes movimientos migratorios, los logopedas se ven cada vez más en la necesidad de atender a la diversidad cultural. Gran parte de las técnicas que usan los logopedas occidentales se basan en valores, creencias y prácticas de la cultura mayoritaria. Para no basarse en estereotipos, los profesionales deben conocer los usos comunicativos y las creencias particulares de las diferentes culturas de sus usuarios para ajustar el trato con ellos y con sus familias dentro y fuera del contexto terapéutico. Algunas dificultades que se diagnostican como lingüísticas pueden deberse a una falta de comprensión de la cultura.

Entre las variables culturales a tener en cuenta para una intervención logopédica efectiva está la diversidad de normas de interacción: normas para la toma de turnos, sobre quién puede hablar al niño y cómo se dirige a él, si se puede adaptar el lenguaje, cómo interpretar las intenciones de los niños en sus enunciados tempranos, las rutinas de juego, la percepción y creencias cobre la edad y discapacidad y los comportamientos no verbales (ej. contacto ocular, gestos) (Crago, 1990).

Preguntar a la familia puede contribuir a evitar estereotipos. Para comprender los usos culturales de cada familia es recomendable tener en cuenta las rutinas diarias. En Estados Unidos, McWilliam (2010) sugiere realizar una entrevista basada en rutinas para la evaluación. Sin embargo, en el Estado Español aún no se ha extendido el uso de este tipo de registros si bien cada vez más profesionales lo incorporan en sus prácticas (de las Heras y Rodríguez, 2015). 
En la terapia, es recomendable introducir temas y objetos de la cultura del niño y buscar alternativas dentro de sus prácticas culturales. Por ejemplo, se ha observado que las madres chinas centran los relatos autobiográficos en las relaciones sociales, mientras que las europeas-americanas lo hacen en las experiencias personales (Wang, 2008).

Para llegar a desarrollar competencias de adaptación a la diversidad cultural es esencial que los investigadores tengan en cuenta esta variedad. En una revisión reciente de la investigación sobre bilingüismo realizada por Hammer et al. (2014), se destaca que sobre 182 artículos, en el 84\% se centran en el inglés como lengua no dominante.

Existen escasos estudios sobre otras lenguas. Según Hammer et al. (2014), la comparación entre resultados resulta difícil debido a la inconsistencia de las definiciones, al no especificar siempre los criterios tenidos en cuenta para etiquetar a los niños como bilingües, ni tampoco la cantidad de exposición, el tipo de bilingüismo ni el estatus socioeconómico de las familias (del que únicamente se informa en un 32\% de los estudios). En gran parte de los trabajos, los niños aprendices de lenguas distintas del inglés son de origen emigrante de baja clase social, por lo que los resultados inferiores en vocabulario y otras habilidades podrían deberse esta circunstancia, como se esperaría de los niños monolingües en la misma situación. Estas variables son difíciles de valorar cuando no siempre se especifica el nivel educativo de los padres (únicamente en el $46 \%$ de los casos referidos), siendo este un predictor de desarrollo bilingüe.

El conocimiento sobre los aspectos culturales y las trayectorias de desarrollo bilingüe y monolingüe por parte de los profesionales que están en contacto con los niños potencialmente bilingües puede contribuir a la mejora en la toma de decisiones y a la optimización de los recursos disponibles.

\section{¿En qué lengua intervenir?}

En varias encuestas publicadas para valorar en qué lengua intervienen y cómo perciben su competencia en la atención a la población multilingüe, los logopedas informan no sentirse competentes (Jordaan, 2007; McLeod, et al., 2013; Williams y Mc Leod, 2012). Actualmente no existe ninguna encuesta de estas características sobre la situación en el Estado Español. Según los resultados de las encuestas disponibles en otros países, los logopedas suelen intervenir en su propia lengua y no siempre en las dos lenguas del niño, aunque en ocasiones pueden servirse de traductores. (Jordaan, 2007, Kritikos, 2003). Las diferentes investigaciones no dejan claro las tareas concretas del traductor en la sesión de evaluación o intervención y, debido a que España no hay 
disponibles aún estudios que recojan este tipo de datos y los servicios de traductores no parecen estar a disposición de los logopedas, no se sabe cuál su función ni la eficacia de la misma. En todo caso, el problema fundamental es el que en ocasiones no se intervenga en la lengua dominante del niño.

Si tomamos como referencia Estados Unidos, la American Speech-LanguageHearing Association (ASHA) considera que el logopeda debe conocer el desarrollo típico en niños bilingües y monolingües, administrar e interpretar una valoración formal e informal y reconocer y comprender la variabilidad y los aspectos culturales que pueden afectar a la interacción terapéutica y familiar. Por otro lado, es recomendable que el logopeda sea competente en las lenguas del niño (ASHA, 2015).

$\mathrm{La} / \mathrm{s}$ lengua/s sobre la/s que se debe intervenir es un tema de debate (GutierrezGlellen, 1999). Se recomienda consultar con los padres la elección de las lenguas y existe un acuerdo de que la lengua hablada en la casa no debe cambiarse (Fredman, 2006). Además, es preferible que la lengua en la que se interviene sea conocida por ambos padres. Idealmente se recomienda intervenir en las dos lenguas del niño y teniendo en cuenta las dos culturas (ASHA, 2015; Gutiérrez-Clellen, 1999; Jordaan, 2007; Kayser, 1995; Thordardottir, 2010). Este tipo de intervención contribuye a un bilingüismo aditivo. También es posible realizar una intervención en la lengua dominante con ayuda de colaboradores, en caso de que el logopeda no sea competente en esta lengua, tratando por otros medios de facilitar el desarrollo de la lengua no dominante.

El objetivo de la intervención será promover cambios funcionales, estimulando la comunicación en entornos significativos para motivar su uso y generalizar y afianzar los aprendizajes. El nivel de competencia adquirido por los niños en ambas lenguas no tiene por qué ser el mismo.

Según la hipótesis de interdependencia (Cummins, 1979) sería necesario un aprendizaje en una primera lengua para desarrollar la segunda lengua. En la práctica, los usos sobre rutinas de la vida diaria son distintos a los de los contextos académicos; las experiencias y los temas de interés en cada lengua pueden ser distintos, por lo que las dos lenguas no tienen por qué compartir un mismo sistema representacional ni transferir todos los aprendizajes. Los entornos significativos se hallan en sus dos lenguas, así como sus experiencias relacionadas con ambas culturas, por lo que la terapia tendría que crear un contexto similar al del niño. Por ejemplo, si el niño tiene sus dos lenguas separadas en función de la escolarización y del contexto familiar, 
convendría tratar sobre los temas escolares en la lengua vehicular de la escuela y sobre los temas cotidianos en la lengua familiar.

La elección de la lengua de intervención no tiene por qué ser una decisión permanente, puede cambiarse con el tiempo y teniendo en cuenta el proceso de recuperación y/o desarrollo de la/s lengua/s en cuestión.

Con respecto a la intervención, varios estudios indican que el enfoque bilingüe siempre es más efectivo (véase revisiones de estos estudios en Kohnert, 2010, Hambly et al. 2013 y Thodardottir, 2010). Thordardottir (2006) defiende que hay que manejar con precaución las prácticas que separan las dos lenguas de forma artificial, como las de no reforzar los intentos comunicativos del niño cuando trata de comunicarse en la lengua que no se está utilizando en la terapia. Esta autora defiende el code-switching (cambio de lengua entre palabras, frases o dentro de las palabras) como uso natural, y considera la comunicación funcional por encima de la lengua utilizada.

Es necesario ampliar la investigación en este campo, para disponer de datos cuantitativos sobre la eficacia del uso de diferentes lenguas en terapia, que permitan tomar decisiones en función de las particularidades de los diferentes contextos.

\section{Opciones de evaluación y eficacia de programas de intervención}

Existen escasos estudios que traten sobre la eficacia de programas de intervención con niños bilingües que presentan alteraciones. Los trabajos disponibles varían según el tipo de intervención, los objetivos clínicos y la población de la muestra (Perozzi y Sánchez, 1992; Schoenbrodt, Kerins y Gesell, 2003; Thordardottir, Weismer y Smith, 1997; Tsybina y Eriks- Brophy, 2010). Los diferentes modelos de intervención tratan de valorar la evolución en el tiempo y/o entre qué lenguas se da una transferencia de lo trabajado en terapia (mejoras en la ejecución en una lengua distinta de aquella en la que se ha intervenido), en el caso de una intervención solo en la lengua dominante o en la lengua no dominante.

A partir de una revisión exhaustiva de investigaciones basadas en la práctica, Thordardottir (2010) resalta que los resultados de los trabajos anteriores muestran un efecto no consistente de la intervención en la lengua dominante como facilitador de la adquisición de la lengua no dominante en todos los aspectos del lenguaje. Se da facilitación, aunque existen diferentes resultados para la generalización a una lengua no dominante, por ejemplo, esta autora encuentra que se generalizan los aprendizajes de 
palabras de uso en el contexto escolar, no así de palabras cotidianas. En este caso los resultados muestran una dependencia del contexto de uso y aprendizaje.

Esta autora también resalta que la intervención sólo en la lengua no dominante no resulta tan efectiva como en la lengua dominante y que los resultados deben medirse en ambas lenguas (Thordardottir et al. 1997). Esto es de especial relevancia en situaciones en las que la lengua dominante es la lengua minoritaria en el contexto social.

En España aún no existen directrices concretas comunes sobre cómo evaluar e intervenir con población bilingüe. En otros países, las organizaciones y asociaciones profesionales, tales como la American Speech-Language-Hearing Association (ASHA, 2015), en Estados Unidos, la Speech-Language and Audiology (SAC), en Canadá, el Royal College of Speech and Language Therapists (RCSLT, 2015), en Reino Unido o en Australia, Speech Pathology Australia, incluyen las guías de buenas prácticas (position papers) (International Expert Panel on Multilingual Children's Speech, 2012).

Estos documentos registran las recomendaciones de las instituciones y asociaciones profesionales y en ellos se marcan las directrices sobre la atención a población multilingüe en cuanto a la formación del logopeda, sus competencias y las particularidades de la atención a la diversidad cultural.

Recientemente se han publicado resultados de una guía de buenas prácticas elaborada por un grupo de expertos en lenguaje de niños multilingües formado por 57 investigadores (logopedas, lingüistas, fonetistas y psicolingüistas) de 33 países, a partir de la valoración del uso profesional de 26 lenguas distintas en la intervención logopédica (McLeod, Verdon y Bowen, 2013). Este documento se basa en la clasificación de la Organización Mundial de la Salud (World Health Organization, 2007). Su objetivo es acordar una terminología y una definición inclusiva de bilingüismo, optando por la definición de Grech y McLeod, (2012), añadiendo la necesidad de considerar dialectos y definiendo a su vez conceptos como "competencia cultural".

Otro objetivo de este documento de buenas prácticas es proponer orientaciones sobre las necesidades de formación, sobre el conocimiento de los patrones de desarrollo bilingüe y los procedimientos de evaluación e intervención más adecuados.

En relación con la evaluación, los expertos informan de la falta de herramientas con baremos multilingües (Hambly et al. 2013), y “culturalmente apropiadas" (McLeod et al. 2013, p.337), ya que en algunas lenguas minoritarias, no existen test ni siquiera para la población monolingüe. A su vez, destacan las dificultades para establecer un 
diagnóstico diferencial entre dificultad y diferencia, para lo que ayudaría tener conocimientos básicos sobre cómo funciona la lengua del sujeto no hablada por el logopeda.

En el abordaje concreto de la evaluación del desarrollo lingüístico en niños bilingües, se ha visto que, debido a la heterogeneidad de la población y la dificultad para estandarizar test para bilingües, se utilizan frecuentemente test baremados con población monolingüe. Su uso puede hacer que se subestimen las capacidades globales del niño, lo que motivaría un sobrediagnóstico de las dificultades (Grimm y Schultz, 2013; Paradis, 2005). Algunas propuestas para organizar la evaluación han sido recogidas por Thodardottir (2010): 1) evaluar al niño en su lengua dominante y compararlo con baremos monolingües. Para ello hay que tener en cuenta que la lengua dominante puede variar con el tiempo, 2) evaluar las dos lenguas y considerar habilidades en conjunto, mediante el uso, por ejemplo, de medidas como el vocabulario total, o 3) evaluar las dos lenguas haciendo un seguimiento. Los cambios en el tiempo y/o la falta de avances puede ser un buen indicador. Con niños menores de 3 años, la observación directa y la entrevista a padres y educadores puede aportar información muy valiosa (Dollaghan y Horner, 2011).

Para registrar cuál es la secuencia de aprendizaje de las lenguas y la lengua dominante y tomar la decisión de la/s lengua/s en la/s que se va a evaluar y el tipo de pruebas, es necesario incluir información adicional en la entrevista para la historia clínica: el país de nacimiento del niño y de los padres, la duración de la residencia en el país y los planes de residencia futuros, la lengua o dialecto utilizada en casa y en el colegio, la lengua en la que se comunica con sus hermanos e iguales, el tiempo de exposición a cada lengua, la competencia de los padres en cada lengua y su nivel de estudios, la edad de adquisición de ambas lenguas por parte del niño y la competencia académica del niño (Rimikis, Smiljanic, y Calandruccio, 2013). El ecomapa es un recurso que puede resultar muy válido para conocer la relación del niño con su entorno. Se trata de un esquema visual que dibuja un profesional con ayuda de la familia y que representa los recursos de apoyo jerarquizados en función de sus fortalezas (McWilliams, 2010). Podría utilizarse para valorar los entornos de uso de cada lengua y los interlocutores habituales.

La evaluación puede incluir una entrevista a los padres, un ecomapa, una entrevista basada en rutinas, pruebas estandarizadas, evaluación dinámica, muestras de lenguaje espontáneo con diferentes interlocutores (teniendo en cuenta los usos de la interacción en las diferentes culturas) y una valoración fonológica de los patrones 
comunes y diferentes en ambas lenguas. Si el logopeda habla la misma lengua que el niño pero no el mismo dialecto, se recomienda que interaccione con él de forma informal durante los 10 minutos previos a la evaluación (Kayser, 1995).

En cuanto a los test estandarizados, estos son susceptibles de modificación. Pueden aceptarse respuestas distintas, realizar cambios en el material, en el contenido o en el tipo de respuestas que se aceptan (Goldstein, 2000). Algunos ejemplos de estas adaptaciones propuestas por Goldstein (2000), son: parafrasear, dar instrucciones adicionales, dar claves, repetir estímulos, proporcionar tiempo extra, saltar ítems no apropiados por temas culturales o de experiencia, pedir aclaraciones o usar puntuaciones alternativas. Por ejemplo, ante un niño bilingüe francés/español, aceptar como correcto el uso de meto por pongo en enunciados como: "meto la comida en la mesa". En el caso de llevar a cabo estas modificaciones hay que tomar nota de las adaptaciones y añadirlas en el informe de evaluación.

Una alternativa para evaluar a niños bilingües son las tareas de evaluación no ligadas a la lengua, como la repetición de pseudopalabras, considerada como un indicador de TEL (Conti-Ramsden, 2003; Roy y Chiat, 2004). Este indicador ha sido inicialmente interpretado como el reflejo de una dificultad de memoria de trabajo (Gathercole y Baddeley, 1990), y considerado posteriormente como una dificultad específica para repetir secuencias multisilábicas nuevas (Archibald, y Gathercole, 2007). Estas tareas han mostrado ser útiles en la evaluación de niños bilingües de español e inglés (Girbau y Schwartz, 2008).

En cuanto a la repetición, las tareas de repetición de frases, también muestran su utilidad como indicador de alteraciones del lenguaje en estas poblaciones bilingües (Chiat et al. 2013).

Asimismo, es imprescindible considerar también la comprensión. En la comprensión de estructuras gramaticales se debe tener en cuenta las características tipológicas de las lenguas. Pham y Kohnert (2010) muestran que, para interpretar una oración como The chair kicked the boy [La silla dio una patada al niño], los niños monolingües de inglés se basan en el orden de palabras, mientras que los niños monolingües de vietnamita lo hacen en la animacidad. Los bilingües de ambas lenguas pueden incluir ambas características. Este tipo de oraciones se encuentran en pruebas estandarizadas como el Test de Comprensión de Estructuras Gramaticales- CEG- - si bien únicamente presenta una versión monolingüe en castellano (Mendoza, Carballo, Muñoz y Fresneda, 2005). 
Por otra parte, las habilidades fonológicas deben evaluarse en todas las lenguas del niño. El inventario fonológico de cada lengua puede llevarnos a marcar como patológica la adquisición de un fonema en diferentes edades. Para ello es necesario manejar este inventario de ambas lenguas y conocer la distribución de los sonidos alófonos. El logopeda debe conocer los patrones fonológicos comunes y atípicos y su orden de adquisición, los patrones dialectales, la dificultad, frecuencia y contextos facilitadores de los sonidos que se trabajarán, así como los errores que más afectan a la inteligibilidad (Yavas y Goldstein, 1998).

Para interpretar la interrelación entre una y otra lengua a nivel fonológico, es importante tener un modelo teórico de partida que condicionará las decisiones sobre la intervención.

Para la intervención en aspectos fonético-fonológicos, el logopeda deberá tratar primero, según la recomendación de Yavas y Goldstein (1998), los patrones que más afecten a la inteligibilidad en ambas lenguas y aquellos con la misma tasa de error en la lengua dominante y la lengua no dominante. En segundo lugar, se tratarán los patrones fonológicos con distinta frecuencia en cada lengua que puedan afectar a la inteligibilidad en ambas lenguas y finalmente se tratarán los patrones fonológicos que afecten a solo una lengua.

Sobre otras patologías y componentes del lenguaje, la literatura es escasa. El Trastorno Específico del Lenguaje (TEL) acapara gran parte de la literatura disponible basada en la evidencia sobre evaluación e intervención logopédica. Destaca un estudio actual sobre niños en edad escolar (Ebert et al. 2014) con 59 niños de 5 a 11 años, bilingües de español-inglés con TEL, cuya lengua dominante es el español, a partir de un diseño de intervención intensiva en tres grupos: 1) con tratamiento sólo en inglés, 2) con tratamiento en inglés y español y 3) con tratamiento en tareas cognitivas no lingüísticas. El último grupo trata de valorar si existe una transferencia entre dominios (específico del lenguaje y general cognitivo) de los logros de la terapia, partiendo de la idea de que aunque los niños con TEL presentan dificultades de procesamiento cognitivo, pueden beneficiarse del tratamiento tanto en habilidades cognitivas generales como en las específicamente lingüísticas. Algunos estudios previos han mostrado resultados positivos de un tratamiento en tareas cognitivas, como rapidez de procesamiento y memoria, en monolingües (Ebert y Kohnert, 2009) y en bilingües (Ebert, Rentmeester-Disher y Kohnert, 2012). 
En Ebert et al. (2014) encontraron que los avances en español en el grupo de tratamiento en inglés no fueron significativos, por lo que deducen que, para niños escolarizados, el tratamiento en una segunda lengua tiene un efecto escaso en la lengua dominante. Los niños del grupo bilingüe, utilizando un $80 \%$ de español en las sesiones, con algunas sesiones de apoyo en inglés por parte de la escuela, mostraron mejoras significativas en inglés, lo que muestra una transferencia de las habilidades adquiridas en la lengua dominante a la lengua no dominante. El tratamiento en tareas cognitivas reveló un efecto en algunas medidas de lenguaje, aunque no en todas. Este resultado abre la posibilidad de una transferencia entre dominios (específico de lenguaje y general cognitivo), aunque no está claro en qué medida se encuentra en la dirección opuesta (si el tratamiento en lenguaje, mejora las habilidades cognitivas), para ello se requiere más investigación.

\section{Orientaciones para el trabajo con familias de niños bilingües con alteraciones del lenguaje}

Además de la intervención logopédica específica, el logopeda tiene la responsabilidad de informar a los padres de los beneficios del bilingüismo y de la posibilidad de mantener ambas lenguas aun cuando al niño se le haya diagnosticado alguna patología del habla y/o el lenguaje. Las preocupaciones de los padres están generalmente relacionadas con el uso de la lengua materna y las opciones de escolarización (De Houwer, 1999b; King y Fogle, 2006). Es importante hacerles entender los beneficios socioculturales y emocionales del uso de dos o más lenguas y que la información disponible de la investigación actual apoya la educación bilingüe. De este modo, aprender más de una lengua no dificulta su desarrollo más allá de las dificultades que ya tienen, ya que "pueden adquirir competencia funcional dentro de los límites de su alteración" (Genesee, 2009, p.9). Los niños bilingües están preparados para aprender una segunda lengua si la exposición es regular y el entorno adecuado. Este aprendizaje requiere un esfuerzo consciente por parte de la familia, para motivar al niño para el aprendizaje de la lengua minoritaria, si es la lengua que se habla en casa y darle oportunidades para hablarla, buscando recursos para multiplicar las experiencias (Pearson, 2010).

Educarse en un ambiente bilingüe no garantiza que un niño sea bilingüe. Aún así, los padres no pueden dejar que sus expectativas dificulten la naturalidad de las 
interacciones. Además tienen que ver la segunda lengua desde el punto de vista de su hijo.

Los padres elegirán un modelo para favorecer el bilingüismo. Este modelo puede ser el de una persona una lengua, tiempo y lugar, separando las lenguas en diferentes momentos y/o entornos, o una estrategia mixta (Pearson, 2010).

En cuanto a la práctica clínica, existen diferentes modelos en función de la implicación de los padres en la terapia. Así: el modelo centrado en el terapeuta, el de los padres como apoyo a la terapia y el centrado en la familia, en los que esta implicación puede ser variable. Hasta la fecha, no existen investigaciones basadas en la evidencia sobre entornos familiares bilingüies que comparen la efectividad de estos modelos entre sí. Watts y McLeod (2009) publicaron un trabajo de recopilación sobre la aplicación modelos con familias de niños con diferentes patologías, concluyendo que la inclusión de la familia en la terapia y/o en la toma de decisiones puede desembocar en resultados positivos.

Debido a la especial importancia del entorno familiar en el desarrollo lingüístico de al menos una de las lenguas del niño bilingüe, frecuentemente en la minoritaria, dependiendo del entorno y del modelo educativo, la intervención logopédica no puede dejar de lado a la familia (de Thorne, 2015). En los casos en los que el logopeda no sea un hablante competente en una de las lenguas, la ayuda de la familia para la transferencia de los aprendizajes entre lenguas y su generalización es fundamental. Por ello, recomendamos valorar cada situación y que tanto el logopeda como la familia orienten las necesidades de intervención logopédica y las aspectos prioritarios de intervención. El objetivo final será que el niño logre alcanzar su máximo potencial como comunicador. Para ello, entrenar a los padres como comunicadores puede ser un punto de partida (Girolametto, Weitzman y Earle, 2013).

\section{Conclusiones}

Los estudios sobre población bilingüe con alteraciones del habla y lenguaje son todavía escasos y más escasos aún en cuanto a los resultados basados en la práctica. A pesar de sus limitaciones, la creciente tendencia a implantar modelos de educación bilingüe y el cambio de actitud de los investigadores, que aún debe extenderse a las instituciones, a los profesionales y a las familias, está promoviendo un interés por los beneficios del bilingüismo, tanto en población típica como en población que presenta alteraciones del 
habla y/o el lenguaje. Ninguna de las investigaciones mencionadas en esta revisión ha mostrado que la intervención en bilingües tenga menor efectividad que en monolingües.

La investigación disponible apoya la postura de que los niños con alteraciones del lenguaje pueden ser bilingües. Si la exposición es adecuada y regular no afectará negativamente a su desarrollo del lenguaje más allá de las limitaciones propias de su patología.

Una vez tomada la decisión de mantener la condición bilingüe de los niños bilingües que presentan alteraciones, surgen dudas y dificultades, entre ellas: ¿son los logopedas competentes para asegurar un avance en todas las lenguas del usuario?, ¿en qué lengua se debe evaluar e intervenir?, ¿de qué herramientas se dispone para su evaluación?, ¿cómo procesan la información los niños bilingües con alteraciones?, ¿cómo es el desarrollo del lenguaje del niño bilingüe?, ¿cuál es el papel de la familia?, ¿cómo se deben manejar las diferencias culturales?, ¿cuáles son los beneficios del bilingüismo a largo plazo?

La evidencia empírica de que disponemos para responder a estas preguntas no es aún lo suficientemente amplia para permitir respuestas definitivas y concluyentes, por lo que la interpretación de los resultados de los estudios disponibles debe ser desde la crítica y analizando detalladamente su perspectiva teórica y la metodología empleada. Para ello, la formación de los logopedas es esencial.

El desarrollo lingüístico de los niños bilingües presenta puntos en común y particularidades en relación con los niños monolingües, y sus beneficios, tanto lingüísticos como cognitivos, son enormes y se mantienen en el tiempo. Para poder profundizar en ello es necesario ampliar la investigación en estos aspectos y en el análisis de las variables del entorno. Se trata especialmente de aumentar los estudios interculturales, en diferentes poblaciones con diferentes patrones de exposición, tipos de bilingüismo, variables cognitivas, de procesamiento fonológico, morfosintáctico, léxico y de lectoescritura, modalidades de dominancia de lengua y de cambios en esta dominancia. También desde la práctica: estudiar los resultados de los modelos educativos bilinguies y probar la eficacia de los diferentes métodos de evaluación; promoviendo el desarrollo necesario de test específicos para bilingües que reflejen sus particularidades de aprendizaje e incluyan baremos adecuados, evaluar la eficacia de distintos programas de intervención y realizar seguimientos longitudinales en bilingües de diferentes lenguas y con diferentes patologías que permitan observar trayectorias de desarrollo. 
Es fundamental partir de una visión no centrista en la que cada patología es una trayectoria posible de desarrollo, no una trayectoria desviada. La atención a las diferencias individuales puede contribuir a dilucidar variables que son claves para el aprendizaje.

La revisión que se ha realizado pretende contribuir a orientar a los profesionales de la Logopedia en la toma de decisiones para la evaluación, intervención y asesoramiento a familias de niños bilingües. Para ello se pone en disposición de los logopedas un resumen de las variables a tener en cuenta para valorar la condición bilingüe de cada persona, decidir en qué lengua/s intervenir (en función del contexto concreto de cada caso y del planteamiento de la intervención) y poder interpretar los avances. Estas variables incluyen la valoración la secuencia de aprendizaje de las lenguas (en qué momento se aprendió cada lengua), si la lengua se utiliza en casa y/o en la escuela, el tiempo y la cantidad de exposición, la competencia lingüística de los interlocutores, entre otras. El objetivo es plantear la conexión entre la investigación desde la Psicolingüística Evolutiva y la investigación aplicada para proponer opciones que permitan una práctica basada en la evidencia que, a su vez, retroalimente la investigación en la práctica.

\section{Referencias}

Aguila, E., Ramón-Casas, M., Pons, F., y Bosch, L. (2007). La medida del léxico productivo inicial: Aplicación de un cuestionario para la población bilingüe. En E. Diez-Itza (Ed.), Estudios de desarrollo del lenguaje y educación. Oviedo: ICE Monografías de Aula Abierta.

American Speech-Language-Hearing Association (2015). Bilingual Service Delivery(Practice Portal). Recuperado el 12 de Enero de 2015. www.asha.org/Practice-Portal/Professional-Issues/Bilingual-Service-Delivery.

Archibald, L. M., y Gathercole, S. E. (2007). Nonword repetition in specific language impairment: More than a phonological short-term memory deficit. Psychonomic Bulletin \& Review, 14(5), 919-924.

August, D. y Shanahan, T. (2006) Developing literacy in second-language learners: report of the national literacy panel on language-minority children and youth. NJ. Laurence Erlbaum Associates.

Barron-Hauwaert, S. (2004). Language strategies for bilingual families: The oneparent-one-language approach (No. 7). Clevedon: Multilingual Matters. 
Bialystok, E., Craik, F. I., y Freedman, M. (2007). Bilingualism as a protection against the onset of symptoms of dementia. Neuropsychologia, 45(2), 459-464.

Bialystok, E. (2011). Reshaping the mind: the benefits of bilingualism. Canadian Journal of Experimental Psychology, 65(4), 229-235.

Bialystok, E., Peets, K., y Moreno, S. (2014). Producing bilinguals through immersion education: Development of metalinguistic awarenss. Applied Psycholinguistics, 35, 177-191.

Bialystok, E. (2015). The impact of bilingualism on cognition. En R. Scott y S. Kosslyn (Eds.), Emerging trends in the social and behavioral sciences. (pp.1-12), NJ: John Wiley and Sons.

Chiat, S., Armon-Lotem, S., Marinis, T., Polisenska, K., Roy, P. y Seeff-Gabriel, B. (2013). The potential of sentence imitation tasks for assessment of language abilities in sequential bilingual children. En: V. Mueller-Gathercole (Ed.), Issues in the assessment of bilinguals. (pp. 56-89), Reino Unido: Multilingual Matters.

Conboy, B.T. y Kuhl, P. K. (2011). Impact of second-language experience in infancy: brain measures on first-and second-language speech perception. Developmental Science, 4, 242-248.

Conboy, B.T., Brooks, R., Meltzoff, A.N., y Kuhl, P.K. (2015). Social interaction in infants'second-language phonetics: An exploration of brain-behavior relations. Developmental Neuropsychology, 40(4), 216-229.

Conti-Ramsden, G. (2003). Processing and linguistic markers in young children with specific language impairment. Journal of Speech, Language, \& Hearing Research, 46, 1029-1037.

Costa, A., Hernández, M., Costa-Faidella, J., y Sebastián-Gallés, N. (2009). On the bilingual advantage in conflict processing: Now you see it, now you don't. Cognition, 113(2), 135-149.

Craik, F. I., Bialystok, E., y Freedman, M. (2010). Delaying the onset of Alzheimer disease bilingualism as a form of cognitive reserve. Neurology, 75(19), 17261729.

Crago, M. (1990). Professional gatekeeping: The multicultural, multilingual challenge. Communiqué, Abril, 10-13.

Cummins, J. (1979). Linguistic interdependence and the educational development of bilingual children. Review of Educational Research, 49(2), 222-251. 
Daniels, K., Toth, J., y Jacoby, L. (2006). The aging of executive functions. En: E. Bialystok y F.I.M. Craik (Eds). Lifespan cognition: Mechanisms of change (pp. 96111). New York: Oxford University Press.

De Houwer, A. (1999a). Two or more languages in early childhood: Some general points and practical recommendations. Centre for Applied Linguistics. Recuperado el 18 de Febrero d 2015 en http://www.ericdigests.org/2000-2/two.htm

De Houwer, A. (1999b). Environmental factors in early bilingual development: The role of parental beliefs and attitudes. En G. Extra y L. Verhoeven (Eds.), Bilingualism and Migration (pp.75-96). Mouton de Gruyter.

De Houwer, A., Bornstein, M. H., y Leach, D. B. (2005). Assessing early communicative ability: A cross-reporter cumulative score for the MacArthur CDI. Journal of Child Language, 32, 735-758.

De Houwer, A. (2007). Parental language input patterns and children's bilingual use. Applied Psycholinguistics, 28(3), 411-424.

De Houwer, A. (2009). Bilingual first language acquisition. Clevedon: Multilingual Matters.

De Houwer, A., Bornstein, M. H., y Putnick, D. L. (2014). A bilingual-monolingual comparison of young children's vocabulary size: Evidence from comprehension and production. Applied Psycholinguistics, 35(6), 1189-1211.

De las Heras, G., y Rodríguez, L. (2015). Guía de intervención logopédica en las dislalias. Madrid: Síntesis.

De Thorne, L. S. (2015). Rethinking environmental influences on child language development. Journal of Communication Disorders, 57, 1-2.

Dollaghan, C. A., y Horner, E. A. (2011). Bilingual language assessment: A metaanalysis of diagnostic accuracy. Journal of Speech, Language, and Hearing Research, 54(4), 1077-1088.

Döpke, S. (1998). Can the principle of'one person-one language be disregarded as unrealistically elitist? Australian Review of Applied Linguistics, 21, 41-56.

Ebert, K. D., Kohnert, K., Pham, G., Disher, J. R., y Payesteh, B. (2014). Three treatments for bilingual children with primary language impairment: Examining cross-linguistic and cross-domain effects. Journal of Speech, Language, and Hearing Research, 57(1), 172-186. 
Ebert, K. D., Rentmeester-Disher, J., y Kohnert, K. (2012). Nonlinguistic cognitive treatment for bilingual children with primary language impairment. Clinical Linguistics \& Phonetics, 26(6), 485-501.

Ebert, K. D., y Kohnert, K. (2009). Non-linguistic cognitive treatment for primary language impairment. Clinical Linguistics \& Phonetics, 23(9), 647-664.

Emmorey, K., Luk, G., Pyers, J. E., y Bialystok, E. (2008). The source of enhanced cognitive control in bilinguals evidence from bimodal bilinguals. Psychological Science, 19(12), 1201-1206.

España. Ley Orgánica 8/2013, de 9 de diciembre, para la mejora de la calidad educativa. Boletín Oficial del Estado, 10 de diciembre de 2013, núm. 295, pp. 97858- 97921.

España. Ley 27/2007, de 23 de octubre, por la que se reconocen las lenguas de signos españolas y se regulan los medios de apoyo a la comunicación oral de las personas sordas, con discapacidad auditiva y sordociegas. Boletín Oficial del Estado, 24 de octubre de 2007, núm. 255, pp. 43251-43259.

Fenson, L., Dale, P. S., Reznick, J.S., Thal, D., Bates, E., Hartung, J. P., Reilly, J. S y Pethick, S. (1993). MacArthur Communicative Development Inventories: User's guide and technical manual. San Diego, CA: Singular Publishing Group.

Fernández Fontecha, A. (2001). Una selección bibliográfica sobre el método AICLE (Aprendizaje Integrado de Conocimientos curriculares y Lengua Extranjera). Contextos educativos: Revista de Educación, 4, 217-239.

Fernández, J. A. G., Delgado, P. S., Herrero, I. M., y Permisán, C. G. (2010). Estudio del sistema y funcionamiento de las aulas de enlace de la Comunidad de Madrid: de la normativa institucional a la realidad cotidiana. Revista de Educación, 3, 473-493.

Fernández, R., y Halbach, A. (2011). Analysing the situation of teachers in the Madrid bilingual project after four years of implementation. Content and Foreign Language Integrated Learning. Contributions to Multilingualism in European Contexts, 241270.

Fredman, M. (2006). Recommendations for working with bilingual children. Multilingual Affairs Commitee IALP. Folia Phoniatrica et Logopaedica, 58, 458464.

Frisoni, G. B., Rossi, R., y Beltramello, A. (2002). The radial width of the temporal horn in mild cognitive impairment. Journal of Neuroimaging, 12 (4), 351-354. 
Gathercole, S. E., y Baddeley, A. D. (1990). Phonological memory deficits in language disordered children: Is there a causal connection? Journal of Memory \& Language, 29, 336-360.

Gathercole, V. C. M., Thomas, E. M. (2009). Bilingual first language development: Dominant language takeover, threatened minority language take-up. Bilingualism: Language and Cognition, 12, 213-237.

Genesee, F. H. (2009). Early childhood bilingualism: Perils and possibilities.Journal of Applied Research on Learning, 2(2), 1-21.

Genesee, F., y Nicoladis, E. (2007). Bilingual acquisition. En E. Hoff y M. Shatz (Eds.), Handbook of language development, (pp. 324-342). Oxford: Blackwell.

Genesee, F. (2006). Bilingual first language acquisition in perspective. En P. McCardle y E. Hoff (Eds.), Childhood bilingualism: Research on infancy through school age, (pp. 45-67). Clevedon, Reino Unido: Multilingual Matters.

Gindis, B. (1999). Language-related issues for international adoptees and adoptive families. International adoption: Challenges and opportunities, 98-107.

Girbau, D., y Schwartz, R. G. (2007). Non-word repetition in Spanish-speaking children with Specific Language Impairment (SLI). International Journal of Language \& Communication Disorders, 42(1), 59-75.

Girolametto, L., Weitzman, E., y Earle, C. (2013). From words to early sentences: Parent-implemented intervention for late-talking toddlers. En L.A. Rescorla y P.S. Dale (Eds), Late Talkers: Language development, interventions, and outcomes (pp 261-282). Baltimore, MD: Paul H. Brookes Publishing Co.

Goldberg, H., Paradis.J. y Crago, M. (2008). Lexical acquisition over time in minority first language children learning English as a second language. Applied Psycholinguistics, 29, 1-25.

Goldstein, B. (2000). Cultural and linguistic diversity resource guide for speechlanguage pathologists. San Diego: Singular.

Goldstein, B. A., y McLeod, S. (2012). Typical and Atypical Multilingual Speech Acquisition. En: S. McLeod y B.A. Goldstein (eds). Multilingual aspects of speech sound disorders in children. (pp. 84-100). Bristol: Multilingual matters.

González i Planas, F. (2002). Era Val d'Aran: Una comunidad lingüística aislada. Revista Philologica Romanica, 2, 76-88. 
Grech, H. y McLeod, S. (2012). Multilingual speech and language development and disorders. En D. Battle (Ed). Communication disorders in multicultural and international populations (4º ed.) (pp. 120-147). St Louis: Elsevier.

Grimm, A., y Schulz, P. (2014). Specific language impairment and early second language acquisition: the risk of over-and underdiagnosis. Child Indicators Research, 7, 821-841.

Grosjean, F. (2010). Bilingual: Life and reality. Boston: Harvard University Press.

Gutiérrez-Clellen, V. (1999). Language choice in intervention with bilingual children. American Journal of Speech-Language Pathology, 8, 291-301.

Hambly, H., Wren, Y., McLeod, S., y Roulstone, S. (2013). The influence of bilingualism on speech production: A systematic review. International Journal of Language \& Communication Disorders, 48(1), 1-24.

Hakutaka, K., y D'Andrea, D. (1992). Some properties of bilingual maintenance and loss in Mexican background high-school students. Applied Linguistics, 13(1), 72-99.

Hammer, C. S., Hoff, E., Uchikoshi, Y., Gillanders, C., Castro, D. C., y Sandilos, L. E. (2014). The language and literacy development of young dual language learners: A critical review. Early Childhood Research Quarterly, 29 (4), 715-733.

Hoff, E., y Rumiche, R. L. (2011). Studying children in bilingual environments.Research methods in child language: A practical guide. En E. Hoff (Ed.), Research methods in child language: a practical guide (pp- 300-316). Nueva York: John Wiley \& Sons.

Hoff, E., Core, C., Place, S., Rumiche, R., Señor, M., y Parra, M. (2012). Dual language exposure and early bilingual development. Journal of Child Language, 39, 1-27.

Hoff, E., y Core, C. (2013). Input and Language Development in Bilingually Developing Children. Seminars in Speech and Language, 34 (4), 215-226.

Huguet, Á. (2004). La educación bilingüe en el Estado español: situación actual y perspectivas. Cultura y Educación, 16(4), 399-418.

Huguet, Á., Chireac, S. M., Janés, J., Lapresta, C., Navarro, J. L., Querol, M., y Sansó, C. (2006). La educación bilingüe: ¿una respuesta al Aragón trilingüe? Revista de Psicodidáctica, 13(2), 13-31.

Huguet, Á., Chireac, S. M., Navarro, J. L., y Sansó, C. (2011). Tiempo de estancia y aprendizajes lingüísticos. El caso de los escolares inmigrantes en Cataluña. Cultura y Educación, 23(3), 355-370. 
Iglesias, A. y Goldstein, B. (1998). Language and dialectal variations. En: J. Bernthal y N. Bankson (Eds). Articulation and phonological disorders. (pp. 148-171). Boston: Allyn \& Bacon.

International Expert Panel on Multilingual Children's Speech (2012). Multilingual children with speech sound disorders: Position paper. Bathurst, NSW, Australia: Research Institute for Professional Practice, Learning and Education (RIPPLE), Charles Sturt University. Recuperado del 17 de Febrero d 2015. http://www.csu.edu.au/research/multilingual-speech/position-paper

Jacobson, P. F., y Schwartz, R. G. (2005). English past tense use in bilingual children with language impairment. American Journal of Speech-Language Pathology, 14(4), 313-323.

Jordaan, H. (2007). Clinical intervention for bilingual children: an international survey. Folia phoniatrica et logopaedica: official organ of the International Association of Logopedics and Phoniatrics (IALP), 60(2), 97-105.

Kayser, H. (1995). Assessment of speech and language impairments in bilingual children. En: H. Kayser (Ed.), Bilingual speech-language pathology: An Hispanic focus (pp. 243-264). San Diego: Singular Publishing Group.

King, K., y Fogle, L. (2006). Raising bilingual children: Common parental concerns and current research. CALdigest Series, 2, 2-3.

Kohnert, K., Windsor, J., y Ebert, K. D. (2009). Primary or "specific" language impairment and children learning a second language. Brain and Language, 109(2), 101-111.

Kritikos, E. P. (2003). Speech-language pathologists' beliefs about language assessment of bilingual/bicultural individuals. American Journal of Speech-Language Pathology, 12(1), 73-91.

Lambert, W. E. (1974). Culture and language as factors in learning and education. En: F. Aboud y R. D. Meade (eds.), Cultural factors to learning (pp.91-122). Bellinghan, Western Washington State College.

MacLeod, C. M. (1991). Half a century of research on the Stroop effect: an integrative review. Psychological Bulletin, 109(2), 163-184.

Martín Rojo, L., y Mijares, L. (2007). "Sólo en español": Una reflexión sobre la norma monolingüe y la realidad multilingüe en los centros escolares. Revista de Educación, $343,93-112$. 
McLeod, S., Verdon, S., Bowen, C. (2013). International aspirations for speechlanguage pathologists' practice with multilingual children with speech sound disorders: Development of a position paper. Journal of Communication Disorders, 46(4), 375-387.

McWilliam, R. A. (Ed.). (2010). Working with families of young children with special needs. Nueva York: Guilford Press.

Mendoza, E., Carballo, G., Muñoz, J., y Fresneda, M. D. (2005). CEG test de comprensión de estructuras gramaticales. Madrid: TEA.

Miller, C. A. (2011). Auditory processing theories of language disorders: Past, present, and future. Language, Speech, and Hearing Services in Schools, 42(3), 309-319.

Ministerio de Educación, Cultura y Deporte (2002). Marco Común Europeo de Referencia para las Lenguas: Aprendizaje, enseñanza y evaluación. Madrid: Secretaría General Técnica del MECD-Subdirección General de Información y Publicaciones y Grupo ANAYA.

Morales, M. (2000). Convivencia, tolerancia y multilingüismo: educación intercultural, en secundaria. Ministerio de Educación, Cultura y Deporte. Madrid: Narcea Ediciones.

Nicoladis, E., Pika, S., y Marentette, P. (2009). Do French-English bilingual children gesture more than monolingual children? Journal of Psycholinguistic Research, $38,573-585$.

Orgassa, A., y Weerman, F. (2008). Dutch gender in specific language impairment and second language acquisition. Second Language Research, 24(3), 333-364.

Paradis, J. (2001). Do bilingual two-year-olds have separate phonological systems? International Journal of Bilingualism, 5(1), 19-38.

Paradis, J. (2005). Grammatical morphology in children learning English as a second language: implications of similarities with Specific Language Impairment. Language, Speech and Hearing Services in the Schools, 36, 172-187.

Paradis, J. (2007). Bilingual children with specific language impairment: Theoretical and applied issues. Applied Psycholinguistics, 28(3), 551-564.

Paul, L. M., Simons, G. F., y Fennig, C. D. (Eds.). 2015. Ethnologue: Languages of the World, Eighteenth edition. Dallas, Texas: SIL International. Online version. Recuperado el 7 de Enero de 2015. http://www.ethnologue.com 
Pearson, B. Z., Fernandez, S. C., y Oller, D. K. (1993). Lexical development in bilingual infants and toddlers: Comparison to monolingual norms. Language learning, 43(1), 93-120.

Pearson, B. Z., y Fernández, S. (1994). Patterns of interaction in the lexical growth in two languages of bilingual infants and toddlers. Language Learning, 44, 617-653.

Pearson, B. Z. (2010). Consigue que tu hijo sea bilingüe. Madrid: Bilingual Readers.

Pearson, B. Z. (2013). Bilingual late talkers versus late talkers who are bilingual. En L. Rescorla y P. Dale (Eds.), Late Talkers. Language Development, interventions and outcomes (pp. 67-87). Baltimore: Brookes pubishing.

Pérez Martin, M. P., Valmaseda Balanzategui, M. V., y Morgan, G. (2014). Sign Bilingual and Co-enrollment Education for Children with Cochlear Implants in Madrid, En M. Marschark, G. Tang y H. Knoors (Eds), Bilingualism and Bilingual Deaf Education (pp.602-645.). Oxford: Oxford University Press.

Perozzi, J., y Sanchez, M. (1992). The effect of instruction in L1 on receptive acquisition of L2 for bilingual children with language delay. Language, Speech and Hearing Services in Schools, 23, 348-352.

Pettito, L. A., Katerelos, M., Levy, B.C., Gauna,K., Tétreault, K., y Ferraro, V. (2001). Bilingual signed and spoken language acquisition from birth: implications for the mechanisms underlying early bilingual language acquisition. Journal of Child Language, 28, 453-496.

Pham, G., y Kohnert, K. (2010). Sentence interpretation by typically developing Vietnamese-English bilingual children. Applied Psycholinguistics, 31(3), 507-529.

Place, S., y Hoff, E. (2011). Properties of dual language exposure that influence 2-yearolds' bilingual proficiency. Child Development, 82(6), 1834-1849.

Räsänen, S.H.M., y Pine, J.M. (2014). Emotional force of languages in multilingual speakers in Finland. Applied Pyscholinguistics, 35, 443-471.

Rescorla, L.A., y Dale, P.S. (2013). (Eds.), Late talkers: Language development, interventions, and outcomes. Baltimore, MD: Paul H. Brookes Publishing Co.

Riemersma A.M.J. y De Vries, S. (2011). (Eds), Some developments with regard to the provisions of trilingual primary education in minority language communities of the European Union. Ljouwert/Leeuwarden, Holanda: Fryske Akademy. 
Rimikis, S., Smiljanic, R., y Calandruccio, L. (2013). Nonnative English speaker performance on the basic English lexicon (BEL) sentences. Journal of Speech, Language, and Hearing Research, 56(3), 792-804.

Ronjat, J., Escudé, P., y Lieutard, H. (1913). Le développement du langage observé chez. un enfant bilingue. Paris: Paris H. Champion.

Roy, P., y Chiat, S. (2004). A prosodically controlled word and nonword repetition task for 2- to 4-year-olds: Evidence from typically developing children. Journal of Speech, Language, \& Hearing Research, 4, 223-234

Royal College of Speech and Language Therapists (RSCLT). (2015). Good practice for speech and language therapists working with clients from linguistic minority communities.

$\begin{array}{lllllll}\text { Recuperado } & \text { el } & 7 & \text { de } & \text { Enero } & \text { de } & 2015\end{array}$ http://www.rcslt.org/members/publications/publications2/linguistic_minorities.

Schoenbrodt, L., Kerins, M., y Gesell, J. (2003). Using narrative language intervention as a tool to increase communicative competence in Spanish-speaking children. Language Culture and Curriculum, 16(1), 48-59.

Skuntnab-Kangas, T. (1981). Bilingualism or not: The education of minorities. Clevedon: Multilingual Matters.

Smithson, L., Nicoladis, E., y Marentette, P. (2011). Bilingual children's gesture use. Gesture, 11(3), 330-347.

Stroop, J. R. (1935). The basis of Ligon's theory. American Journal of Psychology, 47, 499-504.

Teuber, J. F., y Furlong, M. F. (1985). The concurrent validity of the Expressive OneWord Picture Vocabulary Test for Mexican-American children. Psychology in the Schools, 22, 269-273.

Thordardottir, E Weismer, E.S., y Smith, M. (1997). Vocabulary learning in bilingual and monolingual clinical intervention. Child Language Teaching and Therapy, 13, $215-227$.

Thordardottir, E. (2006). Language intervention from a bilingual mindset. The ASHA leader, 11(10), 6-7.

Thordardottir, E., Rothenberg, A., Rivard, M., y Naves, T. (2006). Bilingual assessment: Can overal proficency be estimated from separate measurement of two languages? Journal of Multilingual Communication Disorders, 4, 1-21. 
Thordardottir, E. (2010). Towards evidence-based practice in language intervention for bilingual children. Journal of Communication Disorders, 43(6), 523-537.

Tsybina, I., y Eriks-Brophy, A. (2010). Bilingual dialogic book-reading intervention for preschoolers with slow expressive vocabulary development. Journal of Communication Disorders, 43(6), 538-556.

Wang, Q. (2008). Emotion knowledge and autobiographical memory across the preschool years: A cross-cultural longitudinal investigation. Cognition, 108, 117 135.

Watts, N y Mc Leod, S (2009). Working with families in speech-language pathology. San Diego. Plural Publishing.

Werker, J. F., y Byers-Heinlein, K. (2008). Bilingualism in infancy: First steps in perception and comprehension. Trends in Cognitive Sciences, 12, 144-151.

Williams, C. J., y McLeod, S. (2012). Speech-language pathologists' assessment and intervention practices with multilingual children. International Journal of Speech Language Pathology, 14(3), 292-305.

World Health Organization (WHO Workgroup for development of version of ICF for Children Youth). (2007). International classification of functioning, disability and health: Children and youth version: ICF-CY. Geneva: World Health Organization.

Wright, S. C., y Tropp, L. R. (2005). Language and intergroup contact: Investigating the impact of bilingual instruction on children's intergroup attitudes. Group Processes and Intergroup Relations, special issue on Language, Communication, and Intergroup Relations, 8, 309-328.

Yamamoto, M. (2002). Language use in families with parents of different native languages: an investigation of Japanese non-English and Japanese English families. Journal of Multilingual and Multicultural Development, 23 (6), 531-554.

Yavas, M., y Goldstein, B. (1998). Phonological assessment and treatment of bilingual speakers. American Journal of Speech-Language Pathology, 7(2), 49-60.

Yavas, M., y Goldstein, B. (2006). Aspects of bilingual phonology: The case of Spanish-English bilingual children. En Z. Hua y B. Dodd (Eds). Phonological development and disorders in children: A multilingual perspective. (pp. 265-285). Clevedon: Multilingual Matters. 